\title{
BENZO(A)PIRENO, CARBAMATO DE ETILA E METANOL EM CACHAÇAS
}

\author{
Miriam Solange Fernandes Caruso*, Letícia Araujo Farah Nagato e Janete Alaburda \\ Divisão de Bromatologia e Química, Instituto Adolfo Lutz, Av. Dr. Arnaldo, 355, 01246-902 São Paulo - SP, Brasil
}

Recebido em 30/11/09; aceito em 26/5/10; publicado na web em 24/8/10

\begin{abstract}
BENZO(A)PYRENE, ETHYL CARBAMATE AND METHANOL IN CACHAÇAS. The objective of this work was to evaluate the presence of benzo(a)pyrene (BaP), ethyl carbamate (EC) and methanol in 61 samples of cachaça. The quantification of BaP was carried out using HPLC with fluorescence detection, EC concentrations was determined by GC/MS and that of methanol, by GC/ FID. In all samples, the concentration of methanol remained below $5 \mathrm{mg} 100 \mathrm{~mL}^{-1}$ absolute alcohol. The results of BaP varied from $<0.03$ to $0.86 \mu \mathrm{g} \mathrm{L}^{-1}$; the values of EC exceeded the limit established by Brazilian legislation $\left(150 \mu \mathrm{g} \mathrm{L}^{-1}\right)$ in $53 \%$ of the samples.
\end{abstract}

Keywords: cachaça; sugar cane spirit; organic compounds.

\section{INTRODUÇÃO}

O produto alcoólico obtido a partir da destilação do caldo de cana-de-açúcar fermentado pode ser denominado como aguardente ou cachaça, de acordo com a graduação alcoólica que apresente, conforme a Instrução Normativa n $13 / 2005$, do Ministério da Agricultura Pecuária e Abastecimento (MAPA). A aguardente de cana é a bebida com teor alcoólico de 38 a 54\% v/v, enquanto a cachaça, de 38 a $48 \%$ v/v. ${ }^{1}$ A expressão cachaça só é permitida para a bebida produzida exclusivamente no Brasil ${ }^{2}$ e corresponde a uma das bebidas brasileiras mais populares, com uma produção anual estimada em 1,4 bilhão de litros. Segundo dados do MAPA, apenas em 2006, as exportações de cachaça representaram valores acima de 14 milhões de dólares, os quais vêm aumentando a cada ano. Os maiores compradores são Alemanha, Paraguai, Uruguai, Portugal, Estados Unidos, Argentina e Itália. ${ }^{3}$

As cachaças podem ser produzidas em plantações próprias, colhidas manualmente sem o uso de queimadas e destiladas em alambiques, ou obtidas a partir de grandes culturas, com colheita mecanizada e utilização eventual de queima da palhada e, normalmente, destiladas em coluna. Cerca de $70 \%$ da produção de cachaças corresponde àquelas submetidas a este sistema de destilação. ${ }^{4}$

A ocorrência de contaminantes orgânicos e inorgânicos na cachaça pode ter sua origem na cana-de-açúcar, utilizada como matéria-prima, ou em alguma das diferentes etapas do processo produtivo. Devido ao seu elevado consumo, controlar a qualidade desta bebida é uma questão de saúde pública, portanto, a presença de contaminantes deve ser avaliada. Alguns apresentam elevada toxicidade, dentre eles, o benzo(a) pireno (BaP), carbamato de etila (CE) e metanol. Segundo a legislação brasileira em vigor, ${ }^{1}$ os limites máximos tolerados para o carbamato de etila e metanol, são, respectivamente, $150 \mu \mathrm{g} \mathrm{L}{ }^{-1}$ (ppb) e $20 \mathrm{mg} 100 \mathrm{~mL}^{-1}$ de álcool anidro (AA); ainda não existe limite estabelecido para $\mathrm{BaP}$.

$\mathrm{O} \mathrm{BaP}$, um hidrocarboneto policíclico aromático (HPA) constituído de cinco anéis benzênicos condensados, é considerado pela Agência Internacional de Pesquisas sobre Câncer (IARC) como carcinogênico para humanos (Grupo 1). ${ }^{5}$ Sua formação pode estar associada aos processos de combustão incompleta de matéria orgânica. No caso da cachaça, a queima do canavial durante a fase de colheita pode ser um processo precursor para formação deste composto. Outra forma de contaminação da bebida pode ocorrer pelo contato do caldo da cana com a graxa usada na lubrificação das moendas. Além disso, as resinas asfálticas que revestem os tanques onde podem ser

*e-mail: micaruso@ial.sp.gov.br armazenadas cachaças também podem contribuir para a ocorrência dos contaminantes, pois tanto a graxa como as resinas são fontes naturais de HPAs. ${ }^{6}$

Um outro contaminante presente naturalmente em bebidas alcoólicas e alimentos fermentados é o carbamato de etila (CE), também denominado uretana ou etiluretana. Ele é conhecido há décadas por sua atividade carcinogênica em muitas espécies de animais de laboratório, sendo classificado no grupo $2 \mathrm{~A}$, pela IARC, como provável carcinogênico para humanos. ${ }^{7}$

O carbamato de etila pode ser formado durante todas as etapas de produção das bebidas, pela reação do etanol com alguns compostos nitrogenados, como ureia, carbamil fosfatos, n-carbamil aminoácidos, cianetos, entre outros. Estes compostos são provenientes do próprio meio e como um resultado da atividade microbiológica durante a fermentação alcoólica, sendo a ureia o principal precursor para a formação do CE. ${ }^{8-11}$

O metanol é um álcool presente naturalmente em cachaças em concentrações abaixo de $20 \mathrm{mg} 100 \mathrm{~mL}^{-1}$ de álcool anidro (AA) e sua origem se dá pela hidrólise das pectinas oriundas da matéria-prima. Dependendo da quantidade ingerida, o metanol pode causar intoxicação que se configura inicialmente por dor de cabeça, náusea, cegueira e até a morte. ${ }^{12}$ Os casos de bebidas contaminadas com metanol estão somente relacionados com bebidas alcoólicas clandestinas. ${ }^{13}$

O objetivo deste trabalho foi avaliar a presença de $\mathrm{BaP}$, carbamato de etila e metanol em amostras de cachaça comercializadas na cidade de São Paulo e, também, obtidas diretamente com os próprios fabricantes.

\section{PARTE EXPERIMENTAL}

\section{Amostras}

As amostras de cachaça foram adquiridas em supermercados e lojas especializadas da cidade de São Paulo/SP, Brasil. Foram analisadas um total de 61 amostras de marcas ou lotes diferentes, provenientes dos estados de Minas Gerais (33), São Paulo (22), Pernambuco (2), Ceará (2) e Mato Grosso (2). Das 61 amostras analisadas, 51 eram provenientes de destilação em alambique e 10 em coluna, segundo informações do fabricante.

\section{Materiais}

Os solventes acetonitrila, ciclo-hexano, isopropanol e metanol de grau cromatográfico foram adquiridos da Mallinckrodt-Baker 
(Phillipsburg, EUA). Os reagentes de grau analítico foram adquiridos da Merck (Darmstadt, Germany). A água utilizada para as análises foi purificada num sistema Toraypure Shimadzu (Kyoto, Japan) e filtrada em membrana de nylon de $0,45 \mathrm{~mm}$.

Os padrões de benzo(a)pireno (97\%), de carbamato de etila (99\%) e o de 3-pentanol (98,0\%) foram adquiridos da Sigma (St. Louis, USA), e o de metanol grau cromatográfico (99,5\%), da Merck (Darmstadt, Germany).

\section{Quantificação}

A quantificação dos contaminantes benzo(a)pireno (BaP), carbamato de etila (CE) e metanol presentes nas amostras de cachaças foram realizadas segundo descrito a seguir:

\section{Método I-Benzo(a)pireno}

As análises de $\mathrm{BaP}$ foram feitas segundo o procedimento descrito por Caruso, ${ }^{14}$ utilizando-se um cromatógrafo líquido de alta eficiência (Shimadzu, Kyoto, Japão) composto de degaseificador (DGU-14A), bomba (LC-10AD), detector de fluorescência $\left(\lambda_{\text {exc }} 295\right.$ e $\left.\lambda_{\text {em }} 405 \mathrm{~nm}\right)$ (RF-10A XL), válvula de injeção com loop de $20 \mu \mathrm{L}$ (Rheodyne LP), interface do cromatógrafo com software (CBM 10A) e software Class LC 10. A separação foi realizada em coluna C18 (250 x 4,6 mm, 5 $\mu \mathrm{m})$ da marca Varian (Palo Alto, EUA) e fase móvel: acetonitrila/ água (70:30), fluxo de $1 \mathrm{~mL} / \mathrm{min}$ e a quantificação foi executada por padronização externa com limites de detecção e quantificação de 0,03 e $0,1 \mu \mathrm{g} \mathrm{L}^{-1}$, respectivamente. Para a extração foram utilizados $10 \mathrm{~mL}$ de amostra de cachaça e cartuchos de extração em fase sólida Accubond SPE C-18, 500 mg/6 mL da Agilent (Santa Clara, EUA).

Segundo recomendado para análise de resíduos, foi feita a confirmação da identidade do BaP utilizando-se um sistema cromatográfico (Shimadzu, Kyoto, Japão) composto por cromatógrafo a gás (GC 17A) com detector de massas (QP 5000) e software Class 5000. A separação ocorreu em coluna capilar DB-5MS (30 m x 0,25 mm; $0,25 \mu \mathrm{m}$ ) da J\&W Scientific (EUA), usando-se gás de arraste hélio,

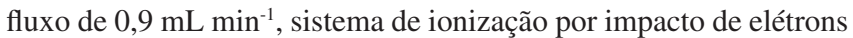
(energia de $70 \mathrm{eV}$ ) e analisador de massas quadrupolo com monitoramento dos fragmentos de massa $(\mathrm{m} / \mathrm{z}) 252$ e 126 . As temperaturas do injetor e detetor foram 250 e $285^{\circ} \mathrm{C}$, respectivamente; a programação térmica do forno foi: $120^{\circ} \mathrm{C}(1 \mathrm{~min}) ; 5^{\circ} \mathrm{C} \min ^{-1}$ até $250^{\circ} \mathrm{C}(0 \mathrm{~min})$; $4{ }^{\circ} \mathrm{C}$ minn $^{-1}$ até $280^{\circ} \mathrm{C}$ (21 min); razão de split: $1: 10 .{ }^{14}$

\section{Método II - Carbamato de etila}

$\mathrm{O}$ CE foi quantificado de acordo com os procedimentos descritos em Nagato et al..$^{15}$ utilizando o mesmo cromatógrafo a gás com detector de massas (CG-MS, Shimadzu, Kyoto, Japão) usado na análise do BaP. A separação ocorreu em coluna capilar FFAP (50 m x $0,25 \mathrm{~mm} ; 0,25 \mu \mathrm{m}$ ) da J\&W Scientific (EUA), usando-se gás de arraste hélio, fluxo de $1 \mathrm{~mL} \mathrm{~min}^{-1}$; sistema de ionização por impacto de elétrons (energia de $70 \mathrm{eV}$ ) e analisador de massas quadrupolo, com monitoramento dos fragmentos de massa $(\mathrm{m} / \mathrm{z}) 62$ e 74 ; volume de injeção de $1,5 \mu \mathrm{L}$; as temperaturas do injetor e detetor foram 220 e $250{ }^{\circ} \mathrm{C}$, respectivamente; a programação térmica do forno foi: 50 ${ }^{\circ} \mathrm{C}(0 \mathrm{~min}) ; 30^{\circ} \mathrm{C} \min ^{-1}$ até $90^{\circ} \mathrm{C}(3 \mathrm{~min}) ; 5^{\circ} \mathrm{C} \min ^{-1}$ até $147^{\circ} \mathrm{C}(0$ min); $20^{\circ} \mathrm{C}$ min $^{-1}$ até $220^{\circ} \mathrm{C}$ (5 min); splitless. A quantificação foi efetuada utilizando-se curva de calibração com padronização interna, empregando-se carbamato de n-propila, e os limites de detecção e quantificação foram 5 e $20 \mu \mathrm{g} \mathrm{L}{ }^{-1}$, respectivamente.

\section{Método III - Metanol}

Para a determinação do metanol foi usado um cromatógrafo a gás, com detector de ionização de chama, de marca Finnigan, modelo 9001 (EUA) e software Borwin, equipado com coluna CP-WAX 52
CB (30m x 0,25 mm; 0,25 $\mu \mathrm{m})$, da Varian (EUA); volume de injeção de $1 \mu \mathrm{L}$. As temperaturas do injetor e detetor foram 210 e $240^{\circ} \mathrm{C}$, respectivamente; a programação térmica do forno foi: $40^{\circ} \mathrm{C}$ ( $\left.25 \mathrm{~min}\right) ; 25$ ${ }^{\circ} \mathrm{C} \min ^{-1}$ até $200{ }^{\circ} \mathrm{C}$ ( 2 min); razão de split: 1:50. A quantificação foi efetuada utilizando-se curva de calibração com padronização interna, empregando-se 3-pentanol, e os limites de detecção e quantificação foram 1 e $5 \mathrm{mg} 100 \mathrm{~mL}^{-1}$ de AA, respectivamente. Este método é também utilizado para determinar as concentrações de componentes secundários em bebidas alcoólicas. ${ }^{16}$

\section{RESULTADOS E DISCUSSÃO}

Os resultados de BaP nas cachaças variaram de não detectado $\left(<0,03 \mu \mathrm{g} \mathrm{L}^{-1}\right)$ a $0,86 \mu \mathrm{g} \mathrm{L}^{-1}$; a Tabela 1 apresenta as faixas de concentração e distribuição de $\mathrm{BaP}$ nas cachaças. Na maioria das amostras, os teores de $\mathrm{BaP}$ situavam-se abaixo do limite de quantificação, que é de $0,10 \mu \mathrm{g} \mathrm{L} \mathrm{L}^{-1}$.

Tabela 1. Faixas de concentração e distribuição de benzo(a)pireno em cachaças, de acordo com o processo de destilação

\begin{tabular}{lccc}
\hline $\begin{array}{l}\text { Faixa de concentração } \\
(\mu \mathrm{g} \mathrm{L})^{-1}\end{array}$ & $\begin{array}{c}\mathrm{N}^{\circ} \text { de } \\
\text { amostras }\end{array}$ & $\begin{array}{c}\text { Destiladas em } \\
\text { alambique }\end{array}$ & $\begin{array}{c}\text { Destiladas em } \\
\text { coluna }\end{array}$ \\
\hline ND a 0,09 & 50 & 45 & 5 \\
0,10 a 0,40 & 9 & 6 & 3 \\
0,41 a 0,86 & 2 & - & 2 \\
\hline
\end{tabular}

ND: não detectado; limite de detecção: $0,03 \mu \mathrm{g} \mathrm{L}^{-1}$; limite de quantificação: $0,10 \mu \mathrm{g} \mathrm{L} \mathrm{L}^{-1}$.

Na literatura, são poucos os trabalhos que relatam a contaminação de hidrocarbonetos policíclicos aromáticos (HPAs) e BaP em bebidas alcoólicas. Tfouni et al. ${ }^{17} \mathrm{em}$ uma pesquisa com 25 amostras de cachaça, obtiveram concentrações de $\mathrm{BaP}$ que variaram de não detectado $\left(<0,011 \mu \mathrm{g} \mathrm{L}^{-1}\right)$ a $0,36 \mu \mathrm{g} \mathrm{L}^{-1}$. Em um estudo semelhante, Bettin e Franco ${ }^{18}$ analisaram 28 amostras de aguardentes e encontraram teores médios de $\mathrm{BaP}$ iguais a não detectado $\left(<0,001 \mu \mathrm{g} \mathrm{L}^{-1}\right)$ para as bebidas produzidas a partir de cana não queimada e a 1,55 $\mu \mathrm{g} \mathrm{L}^{-1}$ para aquelas provenientes de cana queimada. Em outro estudo realizado com 26 amostras de cachaça produzidas com cana queimada e 105 amostras com cana não queimada, verificou-se que as bebidas produzidas a partir de cana queimada apresentaram teores médios totais de HPAs de $21,1 \mu \mathrm{g} \mathrm{L} \mathrm{L}^{-1}$, enquanto que para as outras amostras os teores médios de HPAs totais foram de 1,91 $\mu \mathrm{g} \mathrm{L}^{-1}$. Entretanto, as concentrações médias de $\mathrm{BaP}$ para cachaça de cana queimada, não queimada e envelhecida foram baixas, respectivamente, 0,0456; 0,0168 e $0,0312 \mu \mathrm{g} \mathrm{L}{ }^{-1} \cdot{ }^{19}$

Segundo Novaes, além dos outros fatores prováveis de contaminação de cachaças por HPAs já citados anteriormente, o sistema de aquecimento que ainda é usado por muitas empresas é o vapor que jorra em profusão na base das colunas de aço inoxidável e que é oriundo do acionamento das moendas. Este vapor, geralmente produzido com água não potável, pode arrastar resíduos de HPAs e, consequentemente, contaminar as bebidas. ${ }^{6}$

Com relação ao carbamato de etila, os resultados médios situaram-se na faixa de 20 a $960 \mu \mathrm{g} \mathrm{L}^{-1}$, sendo que em 32 amostras (53\%), os valores estavam acima de $150 \mu \mathrm{g} \mathrm{L}^{-1}$, que é o limite máximo tolerado pelo Ministério da Agricultura, Pecuária e Abastecimento - MAPA, para aguardente de cana e cachaça. Foi estabelecido um prazo de 5 anos para que os fabricantes destas bebidas pudessem se adequar e efetuar o controle em seus produtos. ${ }^{1}$ Esse valor é similar ao definido pelo Departamento de Saúde e Bem Estar do Governo do Canadá que, em dezembro de 1985, fixou os limites de tolerância para carbamato de etila em diversos tipos de bebidas alcoólicas. ${ }^{20}$ 
A Tabela 2 apresenta a distribuição dos teores de carbamato de etila, em $\mu \mathrm{g} \mathrm{L}{ }^{-1}$, por faixas de concentração. Apesar da existência de especificação para este contaminante, foi observado neste estudo que, em grande parte das amostras, os valores de CE foram elevados, ou seja, em 17 cachaças (28\%) a faixa de concentração variou de 150 a $300 \mu \mathrm{g} \mathrm{L}^{-1}$; em 15 cachaças (25\%) foi superior a $300 \mu \mathrm{g} \mathrm{L}^{-1}$, tendo sido encontrados valores superiores a $500 \mu \mathrm{g} \mathrm{L} \mathrm{L}^{-1} \mathrm{em} 6$ amostras.

Tabela 2. Faixas de concentração e distribuição de carbamato de etila em cachaças, de acordo com o processo de destilação

\begin{tabular}{lccc}
\hline $\begin{array}{l}\text { Faixa de concentração } \\
\left(\mu \mathrm{g} \mathrm{L}^{-1}\right)\end{array}$ & $\begin{array}{c}\mathrm{N}^{\mathrm{o}} \text { de } \\
\text { amostras }\end{array}$ & $\begin{array}{c}\text { Destiladas em } \\
\text { alambique }\end{array}$ & $\begin{array}{c}\text { Destiladas em } \\
\text { coluna }\end{array}$ \\
\hline 20 a 150 & 29 & 27 & 2 \\
151 a 300 & 17 & 13 & 4 \\
301 a 960 & 15 & 11 & 4 \\
\hline
\end{tabular}

Limite de detecção: $5 \mu \mathrm{g} \mathrm{L}{ }^{-1}$; limite de quantificação: $20 \mu \mathrm{g} \mathrm{L}^{-1}$.

Aresta et al. estudaram o efeito da presença de precursores para formação do CE nos destilados, como ureia, íons $\mathrm{Cu}$ (II), cianeto e cianato. Foram avaliadas amostras de aguardente de cana e soluções alcoólicas contendo reagentes como sulfato de cobre, cianeto, cianato de potássio e ureia, sob diferentes condições de aquecimento. Nesse estudo foram analisadas 84 amostras de aguardente de cana; a maior parte, $62 \%$, continha teores de CE maiores que $400 \mu \mathrm{g} \mathrm{L}^{-1}$ e somente $13 \%$ das bebidas apresentaram resultados de até $150 \mu \mathrm{g}$ $\mathrm{L}^{-1}$, tendo sido encontrado em uma amostra um teor extremamente elevado, de $5.500 \mu \mathrm{g} \mathrm{L}^{-1}$. Os autores observaram uma correlação positiva entre a concentração de cianeto e a de CE nas amostras de aguardente de cana. ${ }^{21}$

Resultados similares foram encontrados por Nóbrega et al.. Os autores analisaram 25 marcas de cachaça de alambique, oriundas de 19 destilarias do estado da Paraíba; as concentrações de CE para a maioria dessas amostras (70\%) ultrapassaram o limite máximo de $150 \mu \mathrm{g} \mathrm{L}^{-1}$. A faixa de concentração obtida foi de 55 a $700 \mu \mathrm{g} \mathrm{L}^{-1}{ }^{22}$

Bruno e colaboradores estudaram os fatores do processo de destilação que podem ter influência na formação de CE em aguardentes de cana, tais como, material e construção do destilador, tipo de destilação (alambique ou coluna), temperatura e fluxo de destilação, presença de cobre no destilado recém-obtido, presença de precursores nitrogenados no destilado, entre outros. Os autores observaram uma grande dependência da configuração do destilador e do próprio processo de destilação, na formação do CE em cachaças. ${ }^{23}$

Andrade-Sobrinho et al. analisaram, quanto à presença de cobre e CE, 380 amostras de cachaça e 45 de tiquira, comercializadas em várias regiões do Brasil. Nas cachaças destiladas em alambique, os teores médios de $\mathrm{CE}$ foram de $70 \mu \mathrm{g} \mathrm{L} \mathrm{L}^{-1}$, enquanto que nas destiladas em coluna, os valores foram mais elevados, $270 \mu \mathrm{g} \mathrm{L^{-1 }}$. Não foram observadas correlações entre os teores de cobre e de CE, entretanto foi verificada uma tendência de valores de CE mais elevados nas bebidas destiladas em coluna, ${ }^{24}$ isto também foi verificado por Bruno et al..$^{23}$

Labanca e Glória, em um estudo com 71 amostras de aguardente de cana, produzidas e/ou engarrafadas no estado de Minas Gerais, encontraram concentrações de CE ainda mais elevadas do que as obtidas no presente trabalho, as quais variaram de 33 a $2609 \mu \mathrm{g} \mathrm{L}^{-1}{ }^{25}$

O metanol, outro contaminante de elevada toxicidade, também foi avaliado nas 61 amostras de cachaça; a faixa de concentração obtida variou de não quantificado $\left(<5 \mathrm{mg} 100 \mathrm{~mL}^{-1}\right.$ de álcool anidro) a 10,0 mg $100 \mathrm{~mL}^{-1}$ de AA. Todas as bebidas analisadas estavam de acordo com o limite máximo tolerado pela legislação brasileira, que é de $20,0 \mathrm{mg} 100 \mathrm{~mL}^{-1} \mathrm{AA}$.

Os resultados encontrados no presente trabalho foram similares àqueles apresentados por Fernandes et al., em uma pesquisa feita em 16 amostras de cachaça adquiridas diretamente dos respectivos produtores da região sul do Estado de Minas Gerais. ${ }^{26} \mathrm{O}$ mesmo foi observado por Boscolo e colaboradores, em um trabalho realizado com 25 marcas diferentes de cachaça comercializadas em diversas regiões do Brasil. ${ }^{27}$

Apesar dos teores baixos de metanol observados nas amostras analisadas, convém salientar os casos de intoxicação seguida de morte ocorridos no estado da Bahia, em 1989, devido à ingestão de aguardentes contaminadas com metanol, bem como a constatação de 4 casos fatais ocorridos no período de dezembro de 1992 a janeiro de 1993, na região do ABC, na Grande São Paulo. ${ }^{28}$ Em uma pesquisa realizada por Nagato et al. foram analisadas 608 amostras de diversas bebidas alcoólicas no período de abril de 1993 a agosto de 1999. Verificou-se que 391 amostras eram falsificadas e dentre estas amostras, uma aguardente apresentou concentração elevada de metanol, causa da cegueira ocorrida nas pessoas que a consumiram. ${ }^{29}$

O mercado da cachaça é bastante promissor, sendo que a obtenção de bebidas com qualidade superior pode ampliar as fronteiras comerciais e aumentar as exportações, gerando mais divisas e emprego. Desta forma, é necessário exercer um rigoroso controle de qualidade da cadeia produtiva e intensificar a fiscalização, investindo-se também em capacitação técnica para os produtores. Acima de tudo, deve prevalecer a preocupação com a obtenção de bebidas com qualidade, visando a preservação da saúde dos consumidores.

\section{CONSIDERAÇÕES}

A partir dos dados obtidos nas 61 amostras de cachaças, foi possível verificar que benzo(a)pireno e metanol estavam presentes em baixas concentrações; entretanto, o mesmo não ocorreu com o CE, indicando que ainda é necessário melhorar e controlar a qualidade do processo de fabricação deste tipo de bebida, a fim de reduzir a presença deste contaminante e manter os outros em teores tão baixos quanto possível. Devido à carcinogenicidade do carbamato de etila, é preciso assegurar que produtos para o consumo humano, incluindo bebidas alcoólicas, contenham quantidades mínimas desta substância.

Cabe lembrar que ainda não há limite máximo tolerado para $\mathrm{BaP}$ em bebidas alcoólicas; nesse sentido, é importante que mais estudos como este sejam realizados, com o intuito de auxiliar as autoridades competentes no estabelecimento de legislação pertinente.

\section{AGRADECIMENTO}

Suporte financeiro: FAPESP - Fundação de Amparo à Pesquisa do Estado de São Paulo.

\section{REFERÊNCIAS}

1. Brasil, Ministério da Agricultura Pecuária e Abastecimento; Instrução Normativa $n^{\circ} 13$, de 29/6/ 2005, Diário Oficial da União, Seção I, p. 3-3, de 30/06/2005.

2. Brasil, Decreto Federal $n^{\circ} 4062$, de 21/12/ 2001; Diário Oficial da União, Seção I, p. 4, de 26/12/2001.

3. http://www.agricultura.gov.br/portal, acessada em Novembro 2009.

4. http://www.biblioteca.sebrae.com.br, acessada em Novembro 2009.

5. http://monographs.iarc.fr/ ENG/Meetings/92 pahs.pdf, acessada em Novembro 2009.

6. Novaes, F. V.; Engarrafador Moderno 2000, 72, 46.

7. http://monographs.iarc.fr/ENG/Meetings/vol96-summary.pdf, acessada em Novembro 2009.

8. Ough, C. S.; J. Agric. Food Chem. 1976, 24, 323.

9. Battaglia, R.; Conacher, H. B. S.; Page, B. D.; Food Addit. Contam. 1990, 7, 477. 
10. Mackenzie, W. M.; Clyne, A. H.; MacDonald, L. S.; J. Inst. Brew. 1990, 96, 223.

11. Aylott, R. I.; Cochrane, G. C.; Leonard, M. J.; MacDonald, L. S.; Mackenzie, W. M.; McNeish, A. S.; Walker, D. A.; J. Inst. Brew. 1990, 96, 213.

12. Bindler, F.; Voges, E.; Laugel, P.; Food Addit Contam. 1988, 5, 343.

13. Zenebon, O.; Badolato, E. S. G.; Nagato, L. A.; Duran, M. C.; AuedPimentel, S.; Vasconcelos, D. A.; Bol. SBCTA 1996, 30, 71.

14. Caruso, M. S. F.; Alaburda, J.; J. Braz. Chem. Soc. 2009, 20, 502.

15. Nagato, L. A. F.; Silva, O. A.; Yonamine, M.; Penteado, M. D. V. C.; Alimentaria 2000, 311, 31 .

16. Instituto Adolfo Lutz; Métodos físico-químicos para análise de alimentos, $4^{\mathrm{a}}$ ed.; IAL: São Paulo, 2005, cap. 9.

17. Tfouni, S. A. V.; Machado, R. M. D.; Camargo, M. C. R.; Vitorino, S. H. P.; Vicente, E.; Toledo, M. C. F.; Food Chem. 2007, 101, 334.

18. Bettin, S. M.; Franco, D.W.; Cienc. Tecnol. Aliment. 2005, 25, 234.

19. Galinaro, C. A.; Dissertação de Mestrado, Universidade de São Paulo, Brasil, 2006.

20. Conacher, H. B. S.; Page, B. D.; Lau, B. P-Y.; Lawrence, J. F.; Bailey, R.; Calway, P.; Hanchay, J-P.; Mori, B.; J. Assoc. Off. Anal. Chem. 1987, 70, 749 .
21. Aresta, M.; Boscolo, M.; Franco, D. W.; J. Agric. Food Chem. 2001, 49, 2819.

22. Nóbrega, I. C. C.; Pereira, J. A. P.; Paiva, J. E.; Lachenmeier, D. W.; Food Chem. 2009, 117, 693.

23. Bruno, S. N. F.; Vaitsman, D. S.; Kunigami, C. N.; Brasil, M. G.; Food Chem. 2007, 104, 1345.

24. Andrade-Sobrinho, L. G.; Cappelini, L. T. D.; Silva, A. A.; Galinaro, C. A.; Buchviser, S. F.; Cardoso, D. R.; Franco, D. W.; Quim. Nova 2009, $32,116$.

25. Labanca, R. A.; Glória, M. B. A.; Quim. Nova 2008, 31, 1860.

26. Fernandes, W. J.; Cardoso, M. G.; Vilela, F. J.; Morais, A .R.; Silva, V. F.; Nelson, D. L.; J. Food Composit Anal. 2007, 257.

27. Boscolo, M.; Bezerra, C. W. B.; Cardoso, D. R.; Lima Neto, B. S.; Franco, D. W.; J. Braz. Chem. Soc. 2000, 11, 86.

28. Badolato, E. S. G.; Duran, M. C.; Rev. Psiq. Clin. 2000, 27, 1.

29. Nagato, L. A. F.; Duran, M. C.; Caruso, M. S. F.; Barsotti, R. C. F.; Badolato, E. S. G.; Ciênc. Tecnol. Aliment. 2001, 21, 39. 